\title{
Bildgebende Verfahren in der Rheumatologie
}

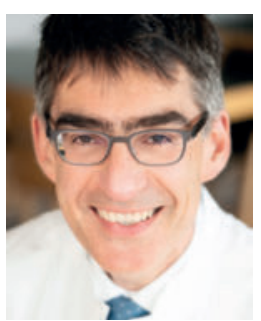

Prof. Dr.

Martin Rudwaleit

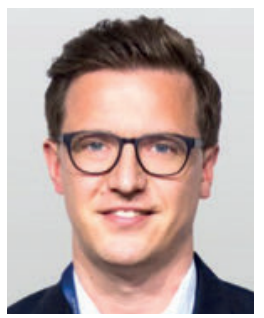

Priv.-Doz. Dr. Philipp Sewerin
Bildgebende Verfahren spielen in der Diagnostik von entzündlich rheumatischen Erkrankungen eine herausragende Rolle. Sie sind nicht selten sogar diagnoseentscheidend und auch für den Therapieverlauf von zunehmender Bedeutung. Während es bis in die 1990er-Jahre vorwiegend das konventionelle Röntgen war, das der Rheumatologe erlernte und beherrschte, so sollte der klinisch tätige Rheumatologe im Jahr 2020 mit verschiedenen bildgebenden Techniken wie der Sonografie, der CT, der MRT und der PET/CT vertraut sein, um Patienten mit entzündlich rheumatischen Erkrankungen adäquat zu managen. Und die Entwicklung neuerer bildgebender Methoden ist damit noch lange nicht zu Ende.

Sich mit den verschiedenen Bildgebungen anzufreunden, ihre Stärken und Limitationen zu kennen und sich selbst in die Lage zu versetzen, die vom Radiologen erstellten Befunde bewerten zu können, stellt eine erhebliche neue Herausforderung an den Rheumatologen dar, unterstreicht andererseits jedoch den Reiz des klinischen Faches Rheumatologie mit den vielen verschiedenen und interessanten Manifestationen und Facetten entzündlich rheumatischer Erkrankungen.

Im vorliegenden Themenheft werden aktuelle Bildgebungsmethoden für den praktisch tätigen Rheumatologen dargestellt. So geht es um den heutigen Stellenwert der konventionellen Röntgendiagnostik bei rheumatoider Arthritis, Spondyloarthritis, bei Arthrosen und Osteoporose. Die MRT der peripheren Gelenke kann hilfreich in der Früherkennung entzündlicher Veränderungen der Synovia, aber auch der Enthesen und des Knochens an Händen und Füßen sein. Die CT kann nicht nur sensitiv ossäre Pathologien abbilden, sondern auch mittels der DECT direkt Uratkristalle detektieren und so bei der Diagnose der Gicht unterstützen.

Abgerundet wird der Themenschwerpunkt durch eine Fallserie von Patientinnen und Patienten mit Großgefäßvaskulitiden und anderen entzündlichen Erkrankungen der großen Gefäße, bei denen die PET-CT die entscheidende Bildgebungsmethode darstellt. In einer der folgenden Ausgaben der arthritis + rheuma werden wir Ihnen außerdem noch ein Update 2020 zur Sonografie bieten.

Viel Freude bei der Lektüre wünschen

Prof. Dr. med. Martin Rudwaleit, Bielefeld

Priv.-Doz. Dr. med. Philipp Sewerin, Düsseldorf 